\title{
Aplicabilidad de criterios internacionales de sostenibilidad para evaluar el diseño urbano de barrios periféricos de origen formal e informal en Bogotá
}

Aplicabilidade de critérios internacionais de sustentabilidade para avaliação do desenho urbano de bairros periféricos de origem formal e informal em Bogotá

Applicability of international sustainability criteria to evaluate the urban design of peripheral districts of formal and informal origin in Bogotá

Juan G. Yunda[a] (1)

[a]Pontificia Universidad Javeriana, Departamento de Arquitectura, Bogotá, Colombia

Cómo citar: Yunda, J. G. (2019). Aplicabilidad de criterios internacionales de sostenibilidad para evaluar el diseño urbano de barrios periféricos de origen formal e informal en Bogotá. urbe. Revista Brasileira de Gestão Urbana, 11, e20180213. https://doi.org/10.1590/2175-3369.011.e20180213.

\section{Resumen}

La teoría internacional del diseño urbano ha propuesto criterios normativos para evaluar el diseño urbano de barrios para asegurar la sostenibilidad integral de los nuevos desarrollos de la ciudad. Estos criterios han sido operados a través de herramientas de evaluación de sostenibilidad como el LEED-ND ${ }^{\circledR}$ estadounidense. A pesar que en Latinoamérica se vienen desarrollando herramientas similares, existen todavía pocas investigaciones que evalúan de manera crítica la aplicabilidad de estos criterios a los diferentes modelos de desarrollo, formal e informal, de barrios locales. Por lo tanto, el presente trabajo intenta contribuir a este debate a través de la aplicación de estos criterios a una muestra de ocho barrios de desarrollo formal e informal en la ciudad de Bogotá. Para realizar esta evaluación crítica, se utilizaron datos tanto cuantitativos a través del uso de SIG, como cualitativos a través de datos de la última encuesta de percepción ciudadana. Los resultados muestran que los criterios son aplicables al entorno latinoamericano ajustando algunos estándares, en diseño de calles, cobertura de transporte público rápido y densidad, pero la metodología de medición no captura las transformaciones informales que pueden contribuir a la sostenibilidad.

Palabras-clave: Sostenibilidad. Urbanismo. Indicadores. Informalidad. Bogotá.

\section{Resumo}

A teoria internacional do design urbano propôs critérios normativos para avaliar o projeto urbano de bairros para garantir a sustentabilidade integral dos desenvolvimentos de novas cidades. Esses critérios foram operacionalizados por meio de ferramentas de avaliação de sustentabilidade, como o americano $L E E D-N D^{\circledR}$. Embora ferramentas semelhantes estejam sendo desenvolvidas na América Latina, ainda existem poucas pesquisas que avaliam criticamente a aplicabilidade desses critérios a diferentes modelos de desenvolvimento formal e informal de vizinhanças locais. Portanto, este trabalho pretende contribuir para esse debate por meio da aplicação desses critérios a uma amostra de oito bairros de desenvolvimento formal e informal, na cidade de Bogotá. Para realizar esta avaliação crítica, foram utilizados tanto dados quantitativos, através do uso de GIS, como dados qualitativos, através de dados da última pesquisa de percepção do cidadão. Os resultados mostram que os critérios

JGY es Doctor en Planificación Regional y de Ciudades, Profesor Asistente, e-mail: yunda.j@javeriana.edu.co 
são aplicáveis ao ambiente latino-americano - ajustando alguns padrões - no desenho de ruas, na cobertura de transporte público rápido e na densidade, mas a metodologia de medição não captura as transformações informais que podem contribuir para a sustentabilidade.

Palavras-chave: Sustentabilidade. Urbanismo. Indicadores. Informalidade. Bogotá.

\section{Abstract}

The international theory of urban design has proposed normative criteria to evaluate the urban design of neighborhoods aiming to ensure integral sustainability of new city developments. These criteria were operationalized through neighborhood sustainability assessment tools, such as the American Leadership in Energy and Environmental Design for Neighborhoods (LEED-ND ${ }^{\circledR}$ ). Although similar tools are being developed in Latin America, studies critically addressing the applicability of these criteria to different models of formal and informal development of local neighborhoods are still scarce. Therefore, this study intends to contribute to this discussion through the application of these criteria to a sample of eight neighborhoods of formal and informal development in the city of Bogotá, Colombia. To this end, both quantitative data from the Geographic Information Systems (GIS) and qualitative data from the latest citizen perception survey were used. Results show that these criteria are applicable to the Latin American environment by adjusting some standards in street design, coverage of rapid public transportation, and density; however, the measurement methodology does not capture the informal transformations that can contribute to sustainability.

Keywords: Sustainability. Urban design. Indicators. Informality. Bogotá.

\section{Introducción}

Durante el siglo XXI el discurso de sostenibilidad ha llegado a todas las disciplinas del desarrollo urbano, desde la gran escala como la planeación regional hasta disciplinas que estudian escalas más pequeñas como el diseño urbano de barrios. De esta manera, el diseño urbano sostenible, o "eco-urbanismo" se ha convertido en el principal paradigma teórico de la disciplina en la actualidad (Sharifi, 2016). Desde la academia norteamericana, este paradigma se ha convertido en la continuación de las corrientes teóricas críticas al urbanismo desarrollado por el Congreso Internacional de Arquitectura Moderna (CIAM). Estas corrientes han evolucionado desde los años 1960, y han llegado a un alto nivel de desarrollo influenciando la praxis del diseño urbano a través de herramientas como los métodos de evaluación de la sostenibilidad de barrios, conocidos en la literatura inglesa como herramientas NSA (Neighborhood Sustainability Assessment), la más conocida de ellas el LEED for Neighborhood Development de Estados Unidos o “LEED-ND ${ }^{\circledR ”}$ (CNU \& USGBC, 2009). En la actualidad esta herramienta ha inspirado métodos similares de evaluación de sostenibilidad de barrios en Latinoamérica como el LEED Brasil Condominio (GBC Brasil, 2017) o el referencial CASA colombiano (CCCS, 2016).

A pesar de esto, en Latinoamérica la transferibilidad de los métodos e indicadores de diseño urbano con origen en morfologías urbanas propias de Norteamérica aún se encuentra en debate (Blanco, 2016; García et al., 2015), y el concepto local de "eco-barrios" esta apenas en una temprana discusión teórica (Valencia et al., 2012; Verdaguer Viana-Cárdenas, 2000). Con base en esto, el presente trabajo intenta contribuir a este vacío evaluando críticamente la aplicabilidad en barrios de Latinoamérica de los criterios normativos de eco-urbanismo propuestos en Norteamérica. Para esto utiliza como caso de estudio ocho barrios periféricos con población de ingresos medios y bajos desarrollados con diferentes modelos de desarrollo en Bogotá. Para esto se utilizaron tanto el análisis de indicadores de morfología urbana obtenidos a través de Sistemas de Información Geográfica (SIG) como los resultados de una encuesta de percepción ciudadana. 


\section{Marco teórico}

En Norteamérica, se entiende hoy como diseño urbano sostenible aquella producción espacial que consigue materializar objetivos de sostenibilidad desde una perspectiva tanto económica, como medioambiental y social simultáneamente (Brundtland, 1987; Campbell, 1996). Es decir, aquel diseño que, si bien produce un desarrollo económico también logra que este se encuentre en armonía con el entorno natural de un lugar, y se encuentra en un marco de justicia social. Este nuevo reto exigió que la teoría del diseño urbano retornara a modelos normativos construidos sobre la base que existe una forma adecuada, o "normativa" de hacer ciudad.

Para esto, los académicos norteamericanos han encontrado en la literatura crítica del CIAM un punto de partida para definir criterios normativos de diseño tanto estéticos, medioambientales y sociales. Es decir, mientras que el CIAM a mediados del siglo XX promovía un diseño urbano y planeación a gran escala, también llamada "comprehensiva", con decisiones de arriba hacia abajo hechas bajo criterios científicos de máximo aprovechamiento de los recursos escasos; teóricos fundacionales de las teorías postmodernas del diseño urbano como Jacobs (1961), Lynch $(1960,1981)$ y Alexander (1966) y Alexander et al. (1977) desarrollaron ideas opuestas. Estos autores revolucionaron la teoría del diseño urbano planteando que las decisiones de ciudad deberían ser consensuadas entre autoridades y comunidades, privilegiando la escala humana y valorando criterios subjetivos como la vitalidad, la identidad y el acceso.

Recientemente en Estados Unidos este discurso "humanista" de los años 1960 a 1980 se ha articulado con emergentes teorías del urbanismo como el diseño ambiental y el neo-tradicionalismo para constituir un cuerpo teórico unificado de eco-urbanismo. En primera instancia académicos como Mumford (1968), Mc Harg (1967) o Spirn (1984), siguiendo la herencia de Geddes (1915), han promovido la integración de la ecología como principio fundamental del diseño urbano. De esta manera el diseño promovido por este "urbanismo ecológico" entiende las ciudades como ecosistemas, hábitats de diferentes especies, conectados y dinámicos (Spirn, 2014) y por ende, produce formas urbanas que respondan como primera medida a los ciclos y contextos ecológicos.

En segunda instancia se encuentra el movimiento de urbanismo neo-tradicionalista que surgió desde la praxis como crítica al diseño urbano enfocado en el automóvil. En la actualidad la ciudad norteamericana crece y está compuesta principalmente por suburbios expandidos de casas unifamiliares junto a grandes superficies comerciales, industriales y de autopistas (Wheeler, 2008). Como crítica a este modelo, basados principalmente en las ideas de Krier (2011), han surgido iniciativas de diseño que propenden por una ciudad más compacta que privilegie la escala humana, la calle y elementos estéticos que estimulen la identidad en las comunidades. Este movimiento es representado principalmente por iniciativas surgidas en la década de 1990 como el "crecimiento urbano inteligente" (Duany et al., 2009), y los principios de diseño del Congreso de Nuevo Urbanismo de Estados Unidos (CNU \& Talen, 1999).

Estos dos movimientos en confluencia han conformado un poderoso cuerpo teórico de urbanismo sostenible (Farr, 2011) que viene influyendo en la práctica del urbanismo en Estados Unidos. Una de las herramientas que han facilitado este proceso son los indicadores de urbanismo sostenible aplicados en las herramientas NSA. Estos indicadores han sido desarrollados dentro del cuerpo teórico mencionado anteriormente para "medir" entornos urbanos reales o planeados de acuerdo a ideales desarrollados desde la teoría, tanto ecológicos como sociales. La herramienta LEED-ND ${ }^{\circledR}$ utiliza las teorías descritas para la formulación de diferentes criterios de evaluación de la morfología urbana. Estos criterios miden la calidad de la localización, morfología y edificios de acuerdo a indicadores base que derivan del contexto urbano norteamericano. Entre los criterios de morfología urbana analizados por el LEED-ND ${ }^{\circledR}$ se encuentran las calles caminables, el desarrollo compacto, la conectividad, la mezcla de usos y tipologías de vivienda, el nivel de acceso a transporte público, espacios verdes y espacios cívicos, la disponibilidad de escuelas barriales, y la participación comunitaria, entre otros.

Estos criterios de morfología urbana empleados en el LEED-ND ${ }^{\circledR}$ coinciden con los planteados por otros autores internacionales. Por ejemplo, Jabareen (2006) en la escala de diseño urbano menciona la 
compacidad, el transporte sostenible, la densidad, los usos mixtos, la diversidad, el diseño solar pasivo y el reverdecimiento como conceptos relacionados con el desarrollo sostenible. Luederitz et al. (2013) encontraron, entre 21 estudios analizados profundamente, nueve principios de desarrollo sostenible de barrios entre los que se destaca el desarrollar sistemas humano-medio ambientales harmónicos y acoplados, el proveer barrios habitables y vibrantes, el desarrollo compacto, la movilidad integrada y sostenible, y el considerar el impacto de los barrios en un entorno más grande. Larco (2016) propuso cinco áreas del diseño urbano sostenible, entre las que se encuentran el uso energético y emisiones, la calidad de agua y recarga, el hábitat y la calidad ecológica, la producción y uso de energía-en temas no enfocados al transporte-la equidad social y la salud.

Esta recopilación de literatura muestra que el norte global ha llegado a un alto grado de consenso acerca de los principios de un diseño urbano sostenible. Sin embargo, la revisión literaria demuestra que estos principios e indicadores son difícilmente transferidos a otros contextos urbanos como el de las ciudades en países en vías de desarrollo. Por ejemplo, la alta densidad es un aspecto problemático. La compacidad y alta densidad es un principio de eco-urbanismo del norte global, que incluso es promovido por importantes entidades multilaterales como la ONU-Hábitat (UN Habitat, 2015, p. 3), sin embargo, diferentes estudios han cuestionado su aplicabilidad en el sur global. Particularmente, Dave (2010) pone en duda la sostenibilidad de la alta densidad en las ciudades de la India ya que encontró asociaciones negativas entre densidad y aspectos como salubridad o satisfacción con el vecindario. Por otro lado, García et al. (2015) encontraron en barrios de altas densidades de Chile menor capital social, sentido comunitario y pertenencia. Finalmente, Boyko \& Cooper (2011) en un análisis de 75 estudios internacionales encontraron correlaciones negativas entre las altas densidades y el caminar por placer, la iluminación natural de las viviendas, y el bienestar psicológico de las personas. Sin embargo, el debate teórico desde el sur global se queda corto en otros aspectos como las calles caminables, la mezcla de usos y tipologías de vivienda, y los diferentes matices de la informalidad en el desarrollo urbano.

Este debate acerca de la aplicabilidad de la densidad y la falta de otros criterios morfológicos de sostenibilidad sobre el diseño de barrios en países en vías de desarrollo es importante en el desarrollo de herramientas similares al LEED-ND ${ }^{\circledR}$ en Latinoamérica, como el caso del LEED Brasil Condominio (GBC Brasil, 2017) y el referencial CASA del Consejo Colombiano de Construcción Sostenible (CCCS, 2016).

Dentro de este marco teórico y el vacío de investigación presentado, el presente trabajo busca incrementar el conocimiento acerca de la pertinencia de los criterios de eco-urbanismo utilizados en el norte global en el contexto latinoamericano. Específicamente se hará énfasis en la morfología de los barrios, ya que es tal vez la mayor diferencia entre los contextos urbanos del norte y el sur global, y en los criterios que parecen no explorar suficientemente las herramientas NSA desarrolladas en la región. El estudio propone para esto el uso de indicadores similares a los que se encuentran en herramientas como el LEED-ND ${ }^{\circledR}$, resumidos en los temas de: Calles Caminables; Desarrollo Compacto y Conectado; Diversidad Social y Acceso a Vivienda; y Mezcla de Usos.

\section{Metodología y caso de estudio}

En cuanto a indicadores de morfología urbana relevantes para la evaluación de barrios se tomaron aquellos indicados en el LEED-ND ${ }^{\circledR}$ y otros estudios que recientemente han intentado medir el diseño urbano de barrios (Adam \& Jamieson, 2014; Ewing \& Handy, 2009; Gehl, 2011; Montero-Serrano et al., 2008; Wheeler, 2015). Estos coinciden en proponer indicadores como la volumetría, alturas, retrocesos, densidades, usos de suelo y conectividad vial como factores para identificar las diferentes morfologías urbanas, y por ende compararlas entre ellas en sus efectos sobre las categorías de eco-urbanismo. Los datos para construir estos indicadores en los barrios de Bogotá fueron tomados de la base de datos espacial Mapa de Referencia v.06.17, disponible en la página web de la oficina de Catastro de la 
municipalidad (UAECD). Estos datos fueron procesados utilizando ArcGIS para determinar diferentes indicadores derivados de la morfología urbana.

De acuerdo con esto, este estudio seleccionó áreas que representan las diferentes morfologías que se han desarrollado en la periferia occidental y sur de la ciudad desde 1980 para una población de ingreso medio-bajo y bajo. Se determinaron estas diferentes morfologías, o tipos de barrios, de acuerdo por la forma de desarrollo y características del crecimiento de cada sector, de una manera similar a la que planteó Yigitcanlar et al. (2015). De acuerdo a esto se proponen cuatro modelos de desarrollo morfológico de barrios en Bogotá entre 1980 a 2015: Proyecto Urbano, Plan Parcial, Normas Mínimas en terreno plano y Normas Mínimas en terreno ladera. Las características de cada uno se presentan en la Tabla 1:

Tabla 1 - Características principales de los modelos de desarrollo propuestos

\begin{tabular}{|c|c|c|c|c|}
\hline $\begin{array}{l}\text { Modelo de } \\
\text { Desarrollo }\end{array}$ & Proyecto Urbano & Plan Parcial & $\begin{array}{c}\text { Normas Mínimas } \\
\text { Plano }\end{array}$ & $\begin{array}{c}\text { Normas Mínimas } \\
\text { Ladera }\end{array}$ \\
\hline Iniciativa & Formal: Pública & Formal: Privada & Informal & Informal \\
\hline Diseño Urbano & $\begin{array}{l}\text { Urbanista } \\
\text { contratado por la } \\
\text { municipalidad }\end{array}$ & $\begin{array}{c}\text { Urbanista } \\
\text { contratado por un } \\
\text { promotor } \\
\text { inmobiliario }\end{array}$ & $\begin{array}{l}\text { No hay urbanista, el } \\
\text { diseño sigue los } \\
\text { parámetros de las } \\
\text { normas mínimas de } \\
\text { urbanización. }\end{array}$ & $\begin{array}{l}\text { No hay urbanista, el } \\
\text { diseño sigue los } \\
\text { parámetros de las } \\
\text { normas mínimas de } \\
\text { urbanización, } \\
\text { condicionado a la } \\
\text { topografía del } \\
\text { terreno. }\end{array}$ \\
\hline Tipo de loteo & $\begin{array}{l}\text { Super-manzanas } \\
\text { para desarrollo por } \\
\text { un promotor } \\
\text { inmobiliario }\end{array}$ & $\begin{array}{l}\text { Super-manzanas } \\
\text { para desarrollo por } \\
\text { un promotor } \\
\text { inmobiliario }\end{array}$ & $\begin{array}{l}\text { Loteo individual para } \\
\text { desarrollo por } \\
\text { autoconstrucción }\end{array}$ & $\begin{array}{l}\text { Loteo individual para } \\
\text { desarrollo por } \\
\text { autoconstrucción }\end{array}$ \\
\hline $\begin{array}{c}\text { Adquisición de la } \\
\text { vivienda }\end{array}$ & $\begin{array}{l}\text { Legalmente con } \\
\text { ahorros familiares y } \\
\text { subsidio de la } \\
\text { municipalidad }\end{array}$ & $\begin{array}{l}\text { Legalmente con } \\
\text { ahorros familiares y } \\
\text { subsidio de la } \\
\text { municipalidad }\end{array}$ & $\begin{array}{l}\text { llegalmente sólo con } \\
\text { ahorros familiares. La } \\
\text { municipalidad } \\
\text { legaliza la titularidad } \\
\text { del predio con } \\
\text { posterioridad. }\end{array}$ & $\begin{array}{l}\text { llegalmente sólo con } \\
\text { ahorros familiares. La } \\
\text { municipalidad } \\
\text { legaliza la titularidad } \\
\text { del predio con } \\
\text { posterioridad. }\end{array}$ \\
\hline
\end{tabular}

Fuente: Elaboración propia.

Teniendo en cuenta estos cuatro modelos se seleccionaron ocho Unidades de Planeación Zonal (UPZ) en Bogotá. Las UPZ son áreas más grandes que los barrios, pero más pequeños que las Localidades. Bogotá está dividida en 117 UPZ las cuales agrupan sectores homogéneos morfológicamente. Debido a su escala y uniformidad la UPZ constituye una escala adecuada para evaluar y comparar las diferentes morfologías de barrios. Las UPZ seleccionadas fueron Bolivia y Tintal Sur como ejemplo de Proyecto Urbano; Calandaima y Las Margaritas como ejemplo de Plan Parcial; Engativá y Patio Bonito como ejemplo de Normas Mínimas en terreno plano; y Lucero y Comuneros como ejemplo de Normas Mínimas en terreno de ladera. El mapa de la Figura 1 muestra la localización de cada UPZ seleccionada en la ciudad con respecto los centros de empleo y a las líneas de transporte rápido de alta capacidad (BRT).

La Figura 2 es un cuadro que proporciona una muestra de la morfología de cada modelo de desarrollo y cada UPZ. En el modelo Proyecto Urbano se observa cómo la forma y tamaño de las manzanas está determinada por un orden estético y un balance entre espacio libre y espacio lleno. En las UPZ del modelo Plan Parcial se observa como las manzanas tienen un tamaño similar, pero no existe una estructura general de proyecto. Esto es causa de un desarrollo fragmentario determinado en mayor medida por la estructura de propiedad de los predios, sin intervención del sector público más allá de exigir un porcentaje de suelo como cesión para áreas verdes y equipamientos. No obstante, tanto para el Proyecto Urbano como para el Plan Parcial la tipología de vivienda es similar, vivienda en serie en bloques multifamiliares de torres de media altura o casas, en ambos modelos, agrupadas en conjuntos cerrados. 
Por otro lado, las de modelo Normas Mínimas tienen una configuración totalmente diferente. En el caso de los terrenos planos las manzanas son pequeñas y alargadas configuradas sobre una retícula regular de calles. En el caso de terrenos de ladera las manzanas tienen un tamaño similar, pero su forma está determinada por las condiciones del terreno. Esto altera la grilla vial y dificulta la conectividad de las calles. Igualmente se observa que en el caso de los barrios planos hay una mayor consolidación de la manzana, en cambio en la ladera, se observan numerosos centros de manzana vacíos que demuestran la dificultad de ocupar el terreno.

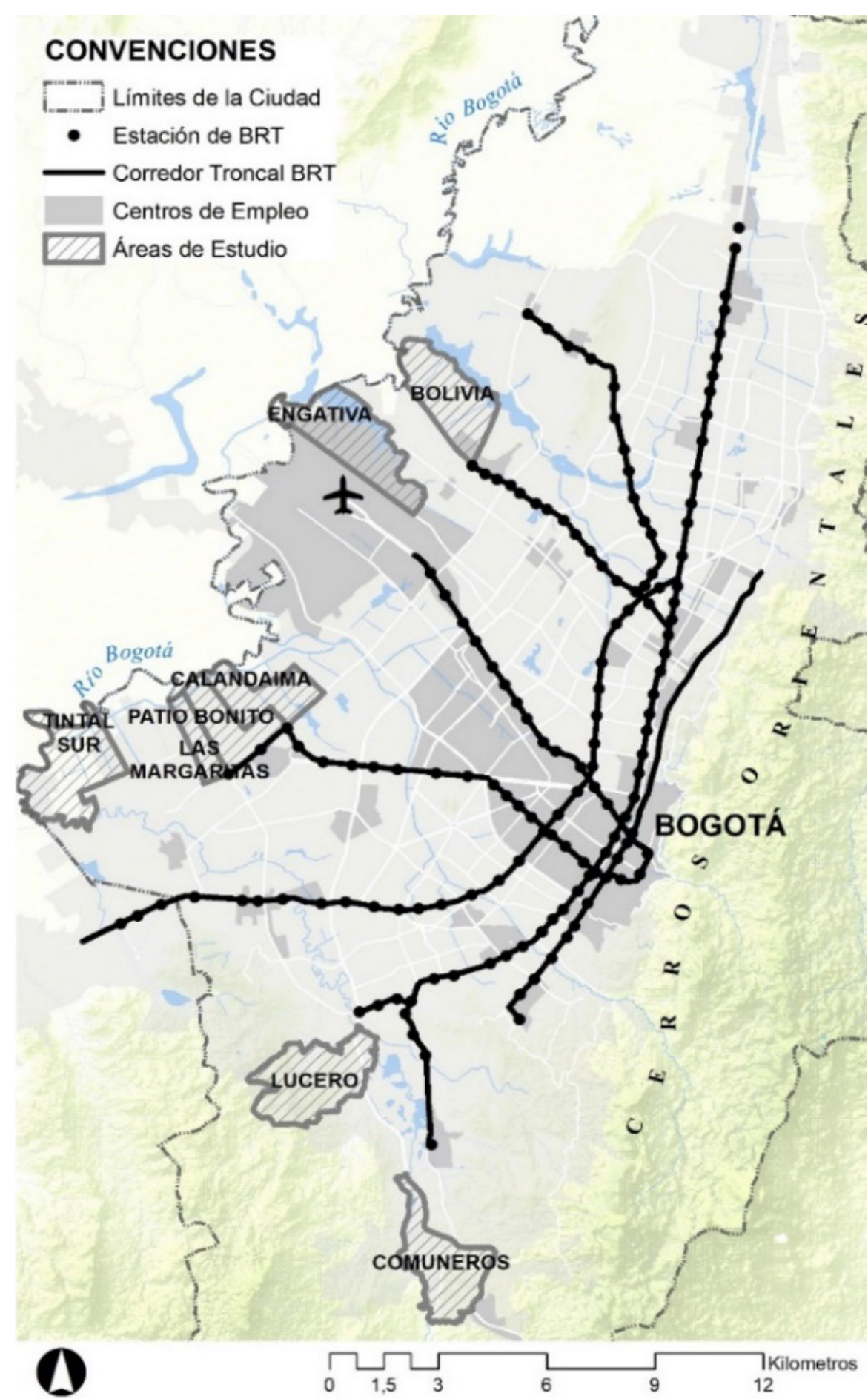

Figura 1 - Localización de las UPZ en Bogotá. Fuente: Elaboración propia con datos del Mapa de Referencia (v.06.17). 

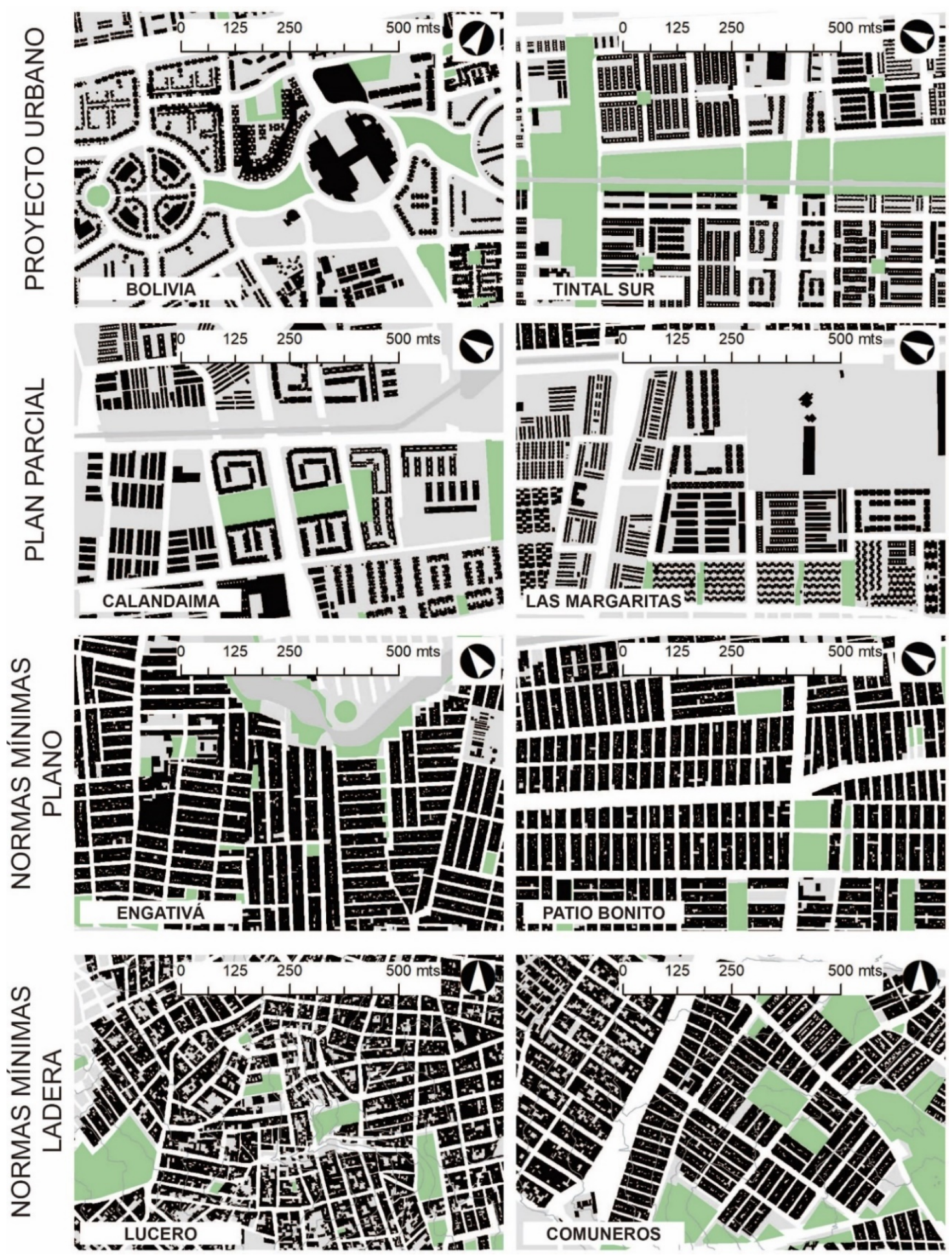

Figura 2 - Muestra de la morfología urbana de cada UPZ y modelo de desarrollo. Fuente: Elaboración propia con datos del Mapa de Referencia (v.06.17).

Con el fin de operacionalizar los indicadores propuestos por las herramientas NSA se proponen cuatro criterios de eco-urbanismo: 1) Calles Caminables; 2) Desarrollo Compacto y Conectado; 3) Diversidad Social y Acceso a Vivienda; y 4) Mezcla de Usos. 
El criterio de Calles Caminables busca proveer entornos donde los peatones sientan comodidad, seguridad y placer al caminar. Si un entorno ofrece estas condiciones se puede reducir el uso del automóvil privado y el consumo de energía, además de incentivar las actividades físicas diarias, mejorando la salud de las comunidades. El segundo criterio de Desarrollo Compacto y Conectado muestra que con suficiente densidad y conectividad un barrio promueve el uso de medios de transporte no motorizados en cortas distancias y soporta adecuadamente el uso del transporte público en distancias largas. El tercer criterio de Diversidad Social y Acceso a la Vivienda en los barrios se enfoca en crear comunidades donde ninguna familia esté excluida por su nivel de ingreso, edad de sus miembros o tamaño. Para esto, se busca que las comunidades ofrezcan una diversidad en la oferta de vivienda, tanto unifamiliares como multifamiliares en edificaciones de diferente altura para ofrecer unidades a distintos precios. Finalmente, el criterio de Usos Mixtos, propone que en los barrios la diversidad de usos incentiva a los habitantes a caminar para acceder a productos y servicios, reduciendo el uso y la dependencia del automóvil privado.

Para medir estos criterios se usaron o adaptaron indicadores sugeridos por el LEED-ND ${ }^{\circledR}$. La Tabla 2 muestra un resumen de los criterios y operaciones espaciales en SIG realizadas.

Tabla 2 - Resumen de los criterios y operaciones espaciales en SIG

\begin{tabular}{|c|c|c|c|c|c|c|}
\hline Criterios & & Variable & $\begin{array}{l}\text { Tipo de } \\
\text { Elemento }\end{array}$ & $\begin{array}{l}\text { Descripción de la } \\
\text { operación } \\
\text { espacial }\end{array}$ & Línea Base & Fuente \\
\hline $\begin{array}{l}\text { Calles } \\
\text { Caminables }\end{array}$ & & $\begin{array}{c}\text { Frentes de } \\
\text { manzana con } \\
\text { accesos directos a } \\
\text { espacio público } \\
\text { Frentes de } \\
\text { manzana con una } \\
\text { proporción de } \\
\text { altura de fachada } \\
\text { a centro de calle } \\
\text { de más de } 1: 1.5\end{array}$ & $\begin{array}{l}\text { Un vector } \\
\text { representa un } \\
\text { tramo vial }\end{array}$ & $\begin{array}{l}\text { Revisión de cada } \\
\text { vector de tramo } \\
\text { vial y clasificación } \\
\text { en (1) si su frente } \\
\text { de manzana tiene } \\
\text { accesos directos a } \\
\text { espacio público, y } \\
\text { (0) si no los tiene. } \\
\text { Revisión de cada } \\
\text { vector de tramo } \\
\text { vial y clasificación } \\
\text { en (1) si su altura } \\
\text { de fachada tiene } \\
\text { una proporción de } \\
\text { más de } 1: 1.5 \text { al } \\
\text { centro de la calle, } \\
\text { y (0) si no la tiene. }\end{array}$ & $\begin{array}{l}90 \% \text { de frentes } \\
\text { de manzana } \\
\text { tienen acceso } \\
\text { directo a } \\
\text { espacio público } \\
\text { sobre la calle. } \\
\\
\text { Máximo el 15\% } \\
\text { de los frentes de } \\
\text { manzana tienen } \\
\text { una proporción } \\
\text { de más de 1:1.5 } \\
\text { al centro de la } \\
\text { calle. }\end{array}$ & $\begin{array}{l}\text { Adaptado de } \\
\text { LEED-ND v. } 4^{\circledR}\end{array}$ \\
\hline \multirow[t]{2}{*}{$\begin{array}{l}\text { Desarrollo } \\
\text { Compacto } \\
\text { Conectado }\end{array}$} & \multirow[t]{2}{*}{ y } & $\begin{array}{l}\text { Densidad neta en } \\
\text { área construida } \\
\text { (viviendas/Ha) }\end{array}$ & $\begin{array}{l}\text { Un polígono } \\
\text { representa una } \\
\text { manzana }\end{array}$ & $\begin{array}{l}\text { Calculo del área } \\
\text { total de los } \\
\text { polígonos que } \\
\text { representan las } \\
\text { manzanas dentro } \\
\text { de los sectores de } \\
\text { estudio. }\end{array}$ & $\begin{array}{c}\text { Pre-requisito: } \\
\text { Más de } 30 \\
\text { viviendas por } \\
\text { hectárea. } \\
\text { Puntos extra a } \\
\text { mayor densidad } \\
\text { hasta un } \\
\text { máximo de } 156 \\
\text { Pre-requisito: } \\
\text { Más de } \\
54 \text { intersecciones } \\
\text { por Km² Puntos } \\
\text { extra a mayores } \\
\text { intersecciones } \\
\text { hasta un } \\
\text { máximo de } 154\end{array}$ & LEED-ND v. $4^{\circledR}$ \\
\hline & & $\begin{array}{l}\text { Intersecciones } \\
\text { externas por } \mathrm{Km}^{2}\end{array}$ & $\begin{array}{l}\text { Un punto } \\
\text { representa una } \\
\text { intersección }\end{array}$ & $\begin{array}{l}\text { Buffer de } 400 \mathrm{~m} \\
\text { alrededor de los } \\
\text { polígonos de los } \\
\text { sectores de } \\
\text { estudio. Suma de } \\
\text { las intersecciones } \\
\text { dentro de estos. }\end{array}$ & $\begin{array}{c}\text { Pre-requisito: } \\
\text { Más de } \\
35 \text { intersecciones } \\
\text { por } \mathrm{Km}^{2}\end{array}$ & LEED-ND v. $4^{\circledR}$ \\
\hline
\end{tabular}


Tabla 2 - Continuación...

\begin{tabular}{|c|c|c|c|c|c|c|}
\hline Criterios & & Variable & $\begin{array}{l}\text { Tipo de } \\
\text { Elemento }\end{array}$ & $\begin{array}{l}\text { Descripción de la } \\
\text { operación } \\
\text { espacial }\end{array}$ & Línea Base & Fuente \\
\hline & & $\begin{array}{l}\text { Cobertura } \\
\text { paradas BRT }\end{array}$ & $\begin{array}{l}\text { Un polígono } \\
\text { representa un } \\
\text { área de } \\
\text { servicio }\end{array}$ & $\begin{array}{l}\text { Análisis de área de } \\
\text { servicio de } 800 \mathrm{~m} \\
\text { de cada parada } \\
\text { de BRT. Cálculo del } \\
\text { número de predios } \\
\text { dentro de esta } \\
\text { área de servicio y } \\
\text { su proporción del } \\
\text { total del sector de } \\
\text { estudio. } \\
\text { Análisis de área de } \\
\text { servicio de } 400 m \\
\text { de cada parada } \\
\text { de bus. Cálculo } \\
\text { del número de } \\
\text { predios dentro de } \\
\text { esta área de } \\
\text { servicio y su } \\
\text { proporción del } \\
\text { total del sector de } \\
\text { estudio. }\end{array}$ & $\begin{array}{l}\text { Pre-requisito: Al } \\
\text { menos } 50 \% \text { de } \\
\text { los predios de } \\
\text { cada sector de } \\
\text { estudio están } \\
\text { dentro de esta } \\
\text { área de servicio. } \\
\\
\text { Pre-requisito: Al } \\
\text { menos } 50 \% \text { de } \\
\text { los predios de } \\
\text { cada sector de } \\
\text { estudio están } \\
\text { dentro de esta } \\
\text { área de servicio. }\end{array}$ & $\begin{array}{l}\text { Adaptado de } \\
\text { LEED-ND v. } 4^{\circledR}\end{array}$ \\
\hline $\begin{array}{l}\text { Diversidad } \\
\text { Social } \\
\text { Acceso } \\
\text { Vivienda }\end{array}$ & $\begin{array}{l}y \\
a\end{array}$ & $\begin{array}{c}\text { Índice Simpson de } \\
\text { Diversidad en } \\
\text { Tipologías }\end{array}$ & $\begin{array}{l}\text { Un polígono } \\
\text { representa un } \\
\text { predio }\end{array}$ & $\begin{array}{c}\text { Cálculo del } \\
\text { número de predios } \\
\text { de cada sector de } \\
\text { estudio en cada } \\
\text { una de las } \\
\text { siguientes } \\
\text { categorías: } \\
\text { Habitacional <4 } \\
\text { pisos en No PH } \\
\text { (Propiedad } \\
\text { Horizontal); } \\
\text { Habitacional <4 } \\
\text { pisos en PH; } \\
\text { Habitacional > 4 } \\
\text { pisos en PH. } \\
\text { Cálculo del índice } \\
\text { Simpson de } \\
\text { diversidad según } \\
\text { estas categorías } \\
\text { para cada sector } \\
\text { de estudio. }\end{array}$ & $\begin{array}{l}\text { Puntos extra a } \\
\text { mayor índice } \\
\text { Simpson desde } \\
0.5 \text { hasta un } \\
\text { máximo de } 0.7 . \\
\text { El Índice Simpson } \\
\text { de Diversidad } \\
\text { (ISD) tiene un } \\
\text { puntaje de cero } \\
\text { a uno, donde } \\
\text { 0.0 demuestra la } \\
\text { existencia de un } \\
\text { solo tipo de } \\
\text { vivienda y } 1.0 \\
\text { muestra un } \\
\text { balance } \\
\text { perfecto entre } \\
\text { todos los tipos } \\
\text { de vivienda } \\
\text { existente. }\end{array}$ & $\begin{array}{l}\text { Adaptado de } \\
\text { LEED-ND v. } 4^{\circledR}\end{array}$ \\
\hline Usos Mixtos & & $\begin{array}{l}\text { Número de usos } \\
\text { de suelo diferentes }\end{array}$ & $\begin{array}{l}\text { Un polígono } \\
\text { representa un } \\
\text { predio }\end{array}$ & $\begin{array}{c}\text { Cálculo del } \\
\text { número de } \\
\text { códigos de uso } \\
\text { diferentes, según } \\
\text { clasificación del } \\
\text { catastro de } \\
\text { Bogotá dentro de } \\
\text { cada sector de } \\
\text { estudio. }\end{array}$ & $\begin{array}{l}\text { Puntos extra al } \\
\text { mayor número } \\
\text { de usos } \\
\text { diferentes desde } \\
4 \text { hasta un } \\
\text { máximo de } 20\end{array}$ & $\begin{array}{l}\text { Adaptado de } \\
\text { LEED-ND v. } 4^{\circledR}\end{array}$ \\
\hline
\end{tabular}

Fuente: Elaboración propia.

Por otro lado, con el fin de contrastar estos indicadores morfológicos con la percepción de los habitantes de los barrios se utilizarán datos secundarios tomados de la Encuesta Multipropósito de Bogotá 2017 (EM-2017) disponibles en la página web del Departamento Administrativo Nacional de Estadística (DANE, 2018). Esta encuesta es el principal ejercicio estadístico de la ciudad que se realiza cada tres años en diferentes zonas con diferentes niveles de ingreso. La EM-2017 es una encuesta por muestreo probabilístico con un tamaño de muestra de 74.640 hogares en Bogotá. Cuenta con una 
precisión esperada en términos de error estándar relativo igual a 7\%, y un nivel de confiabilidad del 95\% para prevalencias de alrededor de 10\%. La encuesta se realizó entre septiembre de 2017 hasta febrero de 2018 con un formulario de 14 capítulos con preguntas a nivel de vivienda, hogar y persona, incluyendo temas como la vivienda y su entorno, condiciones habitacionales, salud, educación entre otros.

\section{Resultados}

Se presenta una descripción socio-económica de cada caso de estudio y los resultados según los criterios propuestos para cada modelo de desarrollo y cada UPZ en la Tabla 3. Los indicadores socio-económicos muestran que, a excepción de Tintal Sur, los barrios de origen formal-Proyecto Urbano y Plan Parcial-son menos poblados, con menor proporción de población pobre y mayor nivel de educación superior que los barrios de origen informal, lo cual es importante al interpretar los resultados.

Tabla 3 - Resultados de indicadores de morfología urbana y percepción de habitantes por UPZ y modelo de desarrollo

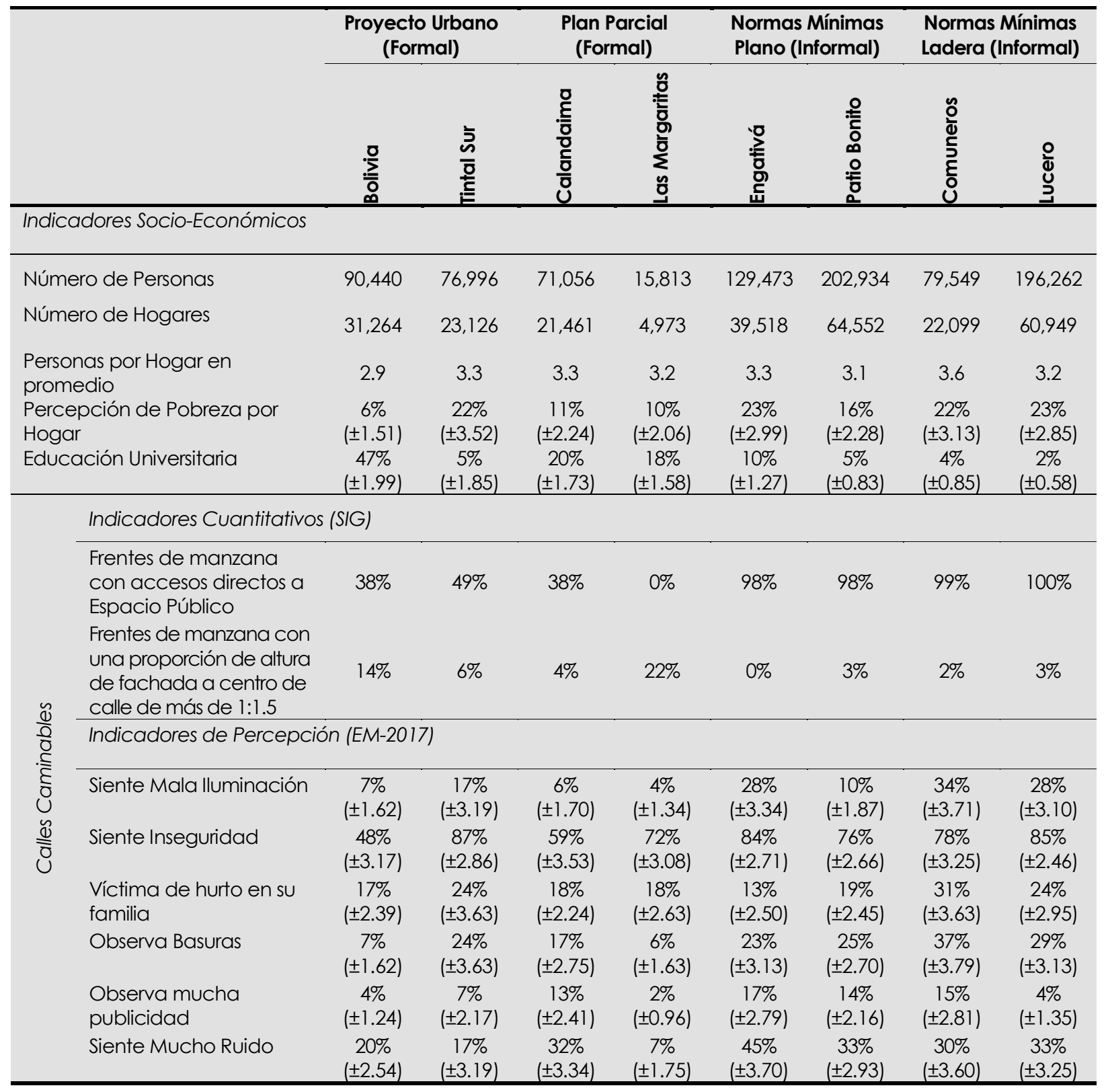


Tabla 3 - Continuación...

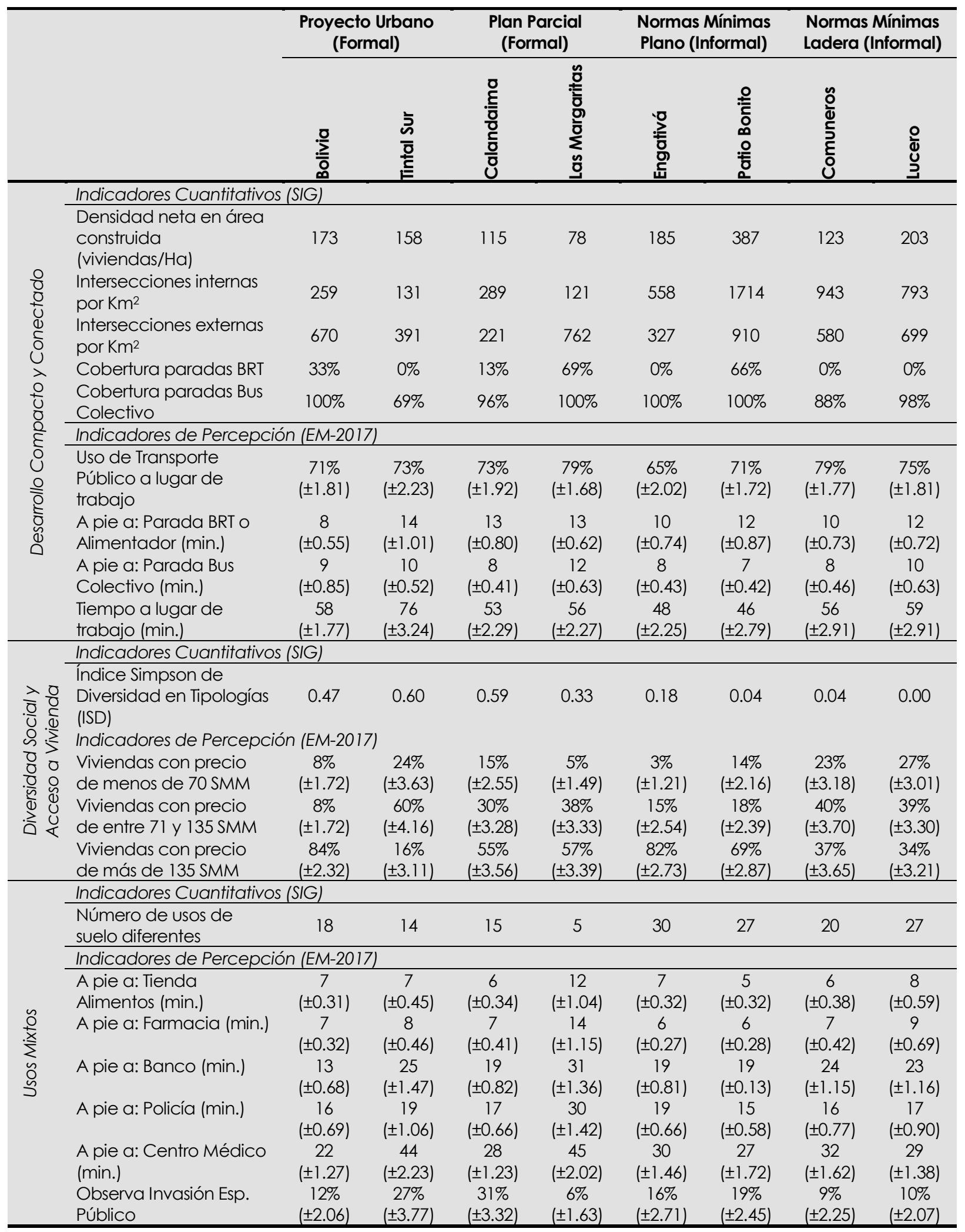

NOTA: Para los resultados de la encuesta en paréntesis se presenta el margen de error a $95 \%$ de confiabilidad. Fuente: Elaboración propia basado en datos de IDECA y EM-2017. 


\section{Calles caminables}

Los datos derivados del análisis SIG indican que las UPZ de origen formal tienen menos del $50 \%$ de los frentes de manzana con acceso directo a espacio público. En su mayoría estas zonas están compuestos de super-manzanas con edificaciones multifamiliares con accesos hacia el interior de la manzana, a áreas de estacionamiento, mientras se cierran al exterior. Estas características, según la teoría norteamericana, sugieren calles que no son caminables ya que frecuentemente se encuentran cerradas por muros ciegos o por largos tramos de cercas ofreciendo un entorno aburrido y sin vigilancia al peatón (ver Figura 3). Sin embargo, en cuanto a calles con escala humana las UPZ de origen formal-a excepción de Las Margaritas-tienen menos de 15\% de frentes de manzana con fachadas con una proporción al centro de la calle mayor a 1:1.5. Esto indica un entorno urbano que ofrece una adecuada escala al peatón, lo que hace más confortable y seguro el caminar.

Por el contrario, en las UPZ de origen informal casi todos los frentes de manzana tienen muchos accesos de viviendas y negocios directos a espacio público y la proporción de la fachada al centro de calle es siempre menor a 1:1.5. Esto facilita que haya más permeabilidad entre el ámbito público y privado, mayor complejidad en las fachadas y mayor vigilancia, haciendo más ameno el caminar. De esta manera ofrecen, según la teoría norteamericana, una mejor condición de calles caminables.

No obstante, los indicadores de percepción muestran un panorama diferente, las UPZ de Proyecto Urbano y Plan Parcial muestran una mayor satisfacción de los habitantes con las calles y el entorno urbano. Por ejemplo, los habitantes perciben mejor iluminación, menor presencia de basuras y publicidad, menos ruido, y sienten levemente mayor seguridad, frente a porcentajes similares de hurtos. El espacio de la calle puede influir en esta percepción, en calles con proporciones de fachada a espacio de calle muy pequeñas las edificaciones están muy cerca de fuentes de ruido y basuras. Esto indica que se podría considerar además de una máxima, una mínima proporción de fachada a centro de calle. Por otro lado, si se comparan los indicadores de percepción de inseguridad, específicamente en los barrios de normas mínimas y en Tintal Sur, esta puede estar también relacionada con la mayor percepción de pobreza en estos barrios.

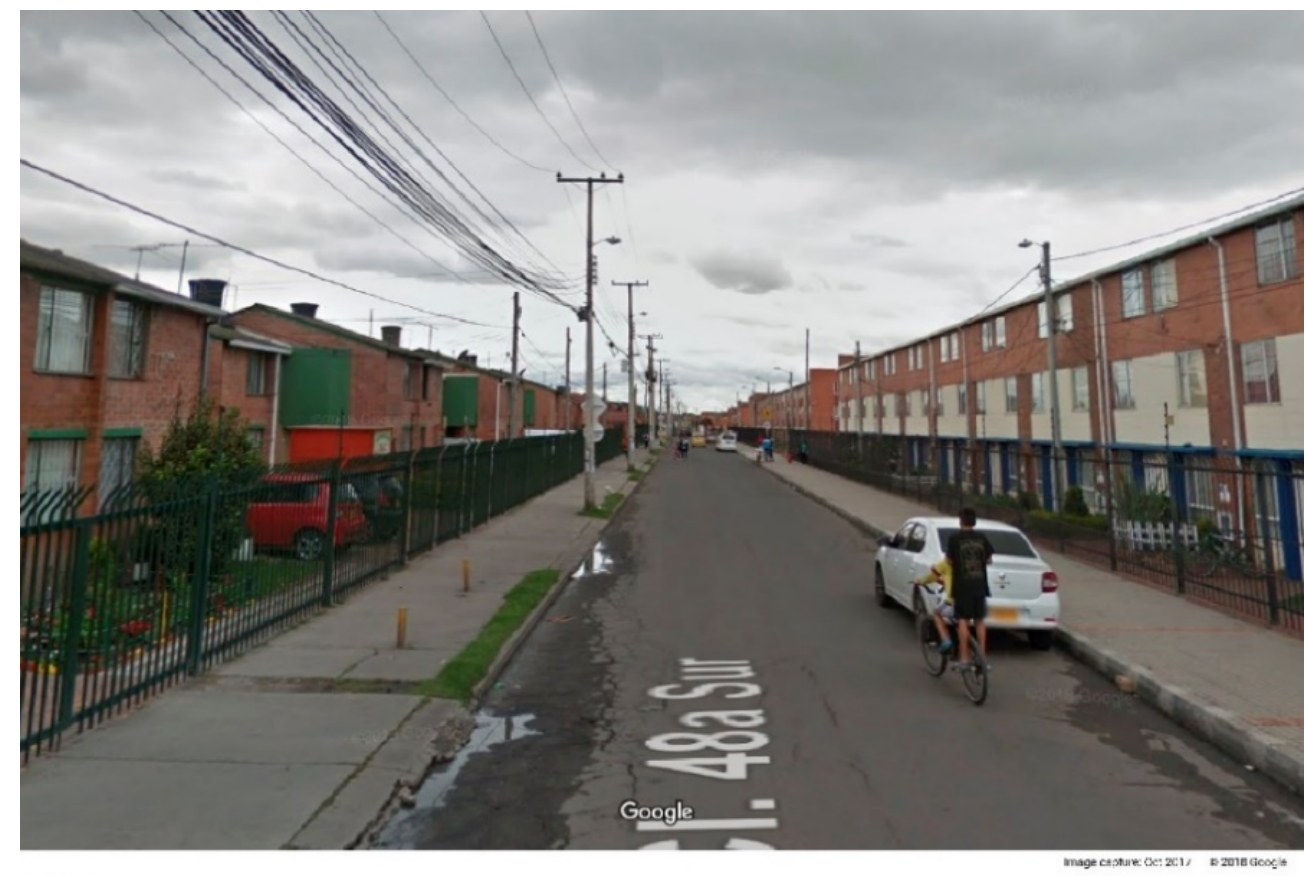

Figura 3 - Largos frentes de manzana sin acceso directo a espacio público en la UPZ Las Margaritas. Fuente: Google Street View. 


\section{Desarrollo compacto y conectado}

En el indicador de densidades sobresale que en general todas las UPZ tanto de origen formal e informal son muy compactas. Estos datos son extremos considerando que el LEED-ND ${ }^{\circledR}$ establece un estándar de mínimo 30 viviendas por hectárea para barrios norteamericanos, y el más alto puntaje se obtiene con densidades mayores apenas a 156 viviendas por hectárea. Esto muestra que los indicadores de base podrían ser mayores para el contexto latinoamericano. Es importante destacar también que las densidades alcanzadas en el Proyecto Urbano, diseñadas por un urbanista, son comparables con las alcanzadas por las áreas de origen informal de Normas Mínimas. Exceptuando el caso de Patio Bonito que alcanza densidades extremas con más de 1.200 habitantes y casi 400 viviendas por hectárea.

El nivel de conectividad de los barrios se mide a través de la cantidad de intersecciones tanto dentro de la UPZ, como del entorno circundante. En este caso el número de intersecciones refleja claramente el modelo de gestión. Las UPZ de origen formal, Proyecto Urbano y Plan Parcial, tienen un nivel de intersecciones internas entre 120 a 290 por kilómetro cuadrado, mientras que en las UPZ de normas mínimas alcanzan todas indicadores superiores a las 500 intersecciones por kilómetro cuadrado. Igual que en el caso de la densidad, estos datos son extremos en comparación con los estándares manejados por el LEED-ND ${ }^{\circledR}$, que tienen como requisito mínimo 54 intersecciones por kilómetro cuadrado y otorgan máximos puntajes a barrios con máximo 154. Los datos de conectividad son incluso mayores si se tiene en cuenta la conectividad de los barrios aledaños, que en la mayoría de veces compensan cuando hay baja conectividad interna. Por ejemplo, en el caso de Las Margaritas, su baja conectividad interna esta compensada con entornos vecinos de origen informal de alta conectividad.

Los datos del análisis SIG también muestran que todas las UPZ tienen una buena cobertura de paradas de buses locales (la mayoría de los predios están a máximo 400 metros de la parada), pero no es igual para las estaciones de BRT donde la mayoría de las UPZ muestran baja cobertura (en solo dos de los casos la mayoría de los predios están a menos de 800 metros de la estación). Esto se confirma con los datos de la encuesta donde los habitantes en todas las UPZ afirman que pueden caminar a una parada de transporte público en menos de 15 minutos, pero al mismo tiempo gastan en la mayoría de los casos más de una hora en llegar a sus trabajos-con el caso extremo de Tintal Sur donde gastan 76 minutos en promedio. Estas cifras muestran que, a pesar de los buenos niveles de compacidad y conectividad, la distancia de cada UPZ con respecto a las estaciones BRT y a los principales centros de empleo puede tener grandes efectos en la calidad de vida de los habitantes.

Llama especialmente la atención los menores tiempos de desplazamiento en las UPZ de Normas Mínimas en terreno plano, Patio Bonito y Engativá. Esto puede responder a que estas UPZ están más próximas al corredor troncal de BRT, pero también al alto porcentaje de usos de suelo no habitacionales. Estos usos frecuentemente incluyen comercios, servicios e industrias que producen puestos de trabajo dentro de los mismos barrios, reduciendo la necesidad de moverse grandes distancias para acceder a oportunidades de empleo (ver Figura 4). 


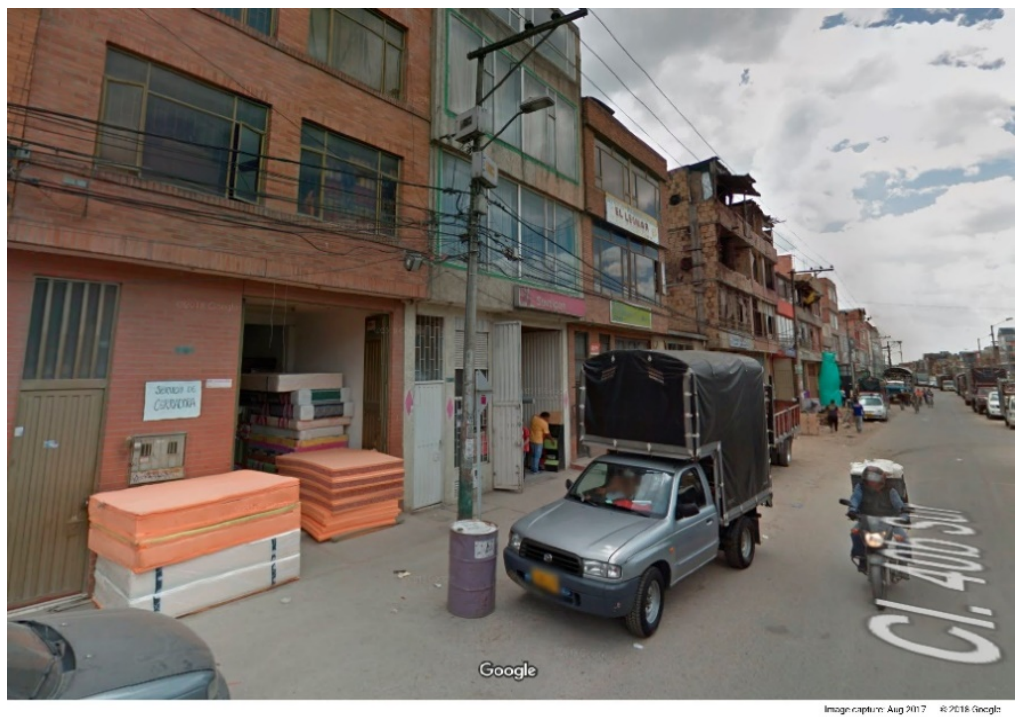

Figura 4 - Usos comerciales e industriales cercanos a la vivienda en UPZ Patio Bonito.

Fuente: Google Street View.

\section{Diversidad social y acceso a vivienda}

Los puntajes de diversidad tipológica según el ISD son muy bajos en las UPZ de Normas Mínimas, tanto en terreno plano como en ladera, y mayores en las UPZ de origen formal, sin embargo, apenas Tintal Sur y Calandaima superan la línea base sugerida por el LEED-ND. Estos bajos puntajes del ISD indican que hay poca diversidad social en la mayoría de casos de estudio. Para validar esta afirmación, el puntaje ISD se contrastó con los rangos de precio de venta de sus viviendas que sugirieron los habitantes. Estos rangos de precio se dividieron en tres categorías: hasta 70 salarios mínimos mensuales (SMM), entre 71 y 135 SMM, y superiores. Esta clasificación coincide con los rangos de precio para aplicar a un subsidio de vivienda en Colombia.

Específicamente, para las UPZ de normas mínimas en ladera, mientras el ISD les da un puntaje muy bajo de diversidad, tienen una variedad de viviendas en los rangos de precio señalados, lo que sugiere que en realidad son muy diversas tipológicamente. Esto puede ser atribuido a la constante transformación informal de las viviendas que difícilmente es capturada por la base de datos SIG. Esta diversidad es validada con indicadores de percepción de pobreza que son los más altos, sugiriendo que ofrecen un alto acceso a la vivienda.

Los casos de origen formal tienen unos indicadores más consistentes donde la diversidad tipológica del ISD se refleja en una relativa diversidad de rangos de precios de vivienda, sin embargo, los porcentajes de vivienda de menor precio son, a excepción de Tintal Sur, menores al 15\%. Esto es validado con los mayores porcentajes de población con educación superior. Esto muestra que en los barrios de origen formal la diversidad social y el acceso a la vivienda por la población más pobre es limitado, con el caso extremo de la UPZ Bolivia, que puede considerarse un "enclave" de clase media.

Es finalmente paradójico también que las UPZ de Normas Mínimas en terreno plano-contrario a las ubicadas en ladera-tengan una sesgada distribución de rangos de precio hacia lo alto, y un bajo ISD por lo que pueden ser también considerados enclaves de clase media. Esto sugiere un patrón de valorización en barrios de origen informal, que no es tenido en cuenta por ningún criterio de eco-urbanismo. 


\section{Usos mixtos}

Para este criterio se evaluó el número de usos no residenciales derivado del SIG y cinco indicadores de percepción sobre acceso a comercio y servicios a pie obtenidos de la EM-2017. Los datos muestran que el número de usos no residenciales en las UPZ de Normas Mínimas en terreno plano y ladera superan los establecidos como máximos por el LEED-ND ${ }^{\circledR}$, y el número de usos en las UPZ de origen formal. Sin embargo, las UPZ de origen formal-a excepción de Las Margaritas-también superan los estándares de eco-urbanismo.

Los datos de la EM-2017 muestran el efecto de los entornos mono-funcionales en la UPZ Las Margaritas. Los habitantes de este barrio señalan mayores tiempos de caminata para acceder a diferentes servicios, gastando casi el doble de tiempo que los habitantes de las otras áreas. Paradójicamente, esto no ocurre en la UPZ Calandaima que tiene una morfología muy similar, pero se presume que esto se debe a los servicios ofrecidos informalmente en el espacio público (ver Figura 5).

En el caso de las UPZ de Proyecto Urbano y las de Normas Mínimas a pesar de la diferencia morfológica y el porcentaje de usos no residenciales, los tiempos promedios que mencionan los habitantes para acceder caminando a comercio y servicios son similares. Esto puede responder a la flexibilidad de la tipología de vivienda autoconstruida en las UPZ de Normas Mínimas, y a la cuidadosa localización de locales de comercio y servicios por parte de un urbanista en el caso de las UPZ de Proyecto Urbano.

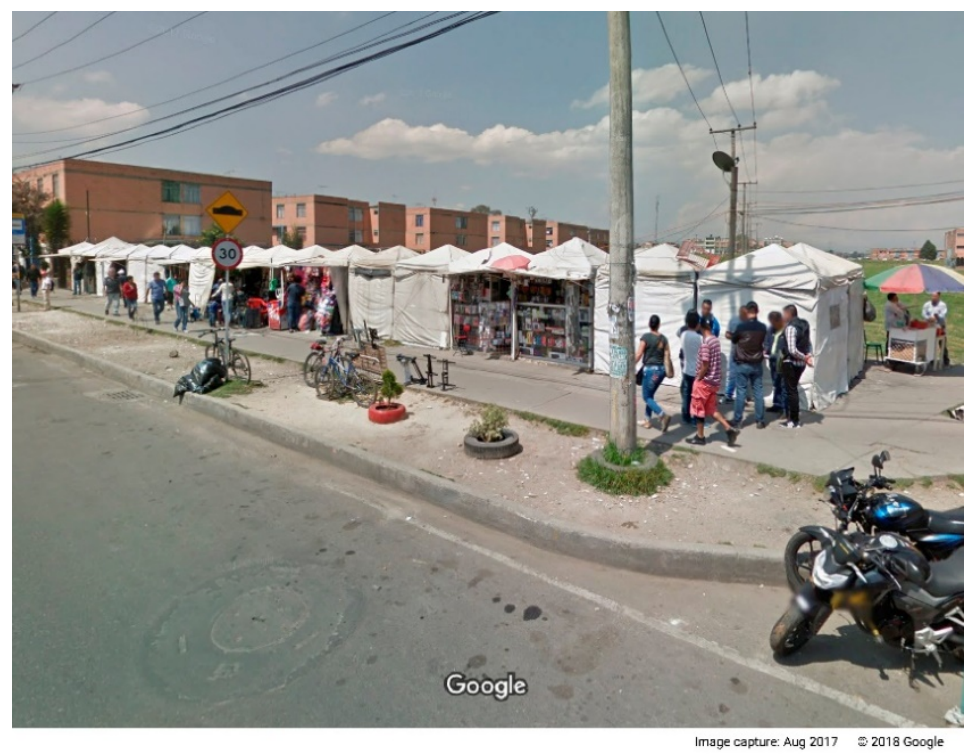

Figura 5 - Mercado informal en UPZ Calandaima. Fuente: Google Street View.

\section{Conclusiones y discusión}

El propósito de este trabajo es analizar la aplicabilidad de los criterios de eco-urbanismo internacionales en el contexto Latinoamericano-Calles Caminables, Desarrollo Compacto y Conectado, Diversidad Social y Acceso a la Vivienda, y Usos Mixtos. Para esto se utilizó la ciudad de Bogotá como caso de estudio, y específicamente, se aplicaron los criterios a ocho barrios periféricos con cuatro diferentes modelos de desarrollo y morfología, con población de ingresos medio-bajos y bajos. Los resultados muestran que los criterios son aplicables al entorno latinoamericano ajustando algunos estándares, pero la metodología de medición no captura la influencia de la informalidad en las transformaciones en vivienda y espacio público que puede contribuir a la sostenibilidad. Se evidencia que los casos de estudio-excepto Las Margaritasson en general sostenibles medidos con la metodología internacional, sin embargo, se evidencian retos en el diseño de calles, diversidad social y acceso a transporte público rápido. 
Específicamente, el criterio de Calles Caminables se observa que los barrios de origen formal ofrecen calles con una adecuada escala humana, pero con muchos frentes de manzana sin permeabilidad. Los barrios de origen informal tienen una alta permeabilidad, pero los resultados de percepción indican que la proporción entre altura de fachadas y ancho de calle puede llegar a ser muy pequeña llevando a incomodidades por ruido, publicidad y basuras. En este caso los criterios internacionales son útiles para identificar los problemas de las calles, pero no existe una línea base mínima de proporción entre altura de fachada y ancho de calle aplicable a los barrios de origen informal.

En cuanto a Desarrollo Compacto y Conectado los resultados muestran que todos los barrios analizados son muy compactos, tanto los barrios de origen formal como informal. Todos los barrios también están muy bien conectados, tienen muchas intersecciones por unidad de área, y cuando las conexiones internas son pocas se compensan con una buena conectividad externa. Igualmente tienen buena cobertura de paradas de buses. Esto se refleja en el intenso uso del transporte público en todos los casos. Sin embargo, la cobertura del transporte público rápido de alta capacidad (BRT) es baja en la mayoría de los barrios, lo que se refleja en los altos tiempos de desplazamiento. En consecuencia, esta cobertura debería enfatizarse en indicadores para el entorno local. En el caso de Patio Bonito que alcanza densidades extremas y alto costo de la propiedad, este estudio llama la atención a si se deben considerar también estándares de densidades máximas.

En el criterio de Diversidad Social y Acceso a la Vivienda los resultados muestran una situación compleja y diferenciada de acuerdo al modelo de desarrollo. Los barrios de origen formal tienen muy poca diversidad social, y pueden ser considerados enclaves tanto de población de clase media o media-baja, mientras la población muy pobre tiene pocas oportunidades de acceder a estos territorios. Por otro lado, los barrios informales en ladera son muy diversos socialmente, una diversidad que no es capturada por los indicadores cuantitativos debido a que las viviendas son de autoconstrucción y cambian en el tiempo. Sorprendentemente, los barrios de origen informal en terreno plano no resultaron socialmente diversos en este estudio, esto puede ser un indicio de extrema valorización, por ello es necesario un análisis más profundo de estos casos.

Finalmente, en el criterio de Usos Mixtos los resultados son positivos en la mayoría de los casos. Esto puede ser obvio en los barrios de origen informal ya que la naturaleza flexible de las edificaciones permite trasformaciones a otros usos diferentes a vivienda. En los barrios de origen formal se encuentran resultados mezclados. En Proyecto Urbano se evidencia la influencia de un urbanista profesional que inserta cuidadosamente usos comerciales y equipamientos en el tejido de vivienda. En los Planes Parciales esto no sucede, pero llama la atención cómo la informalidad suple esta deficiencia de diseño.

\section{Agradecimentos}

Este trabajo fue financiado por la Pontificia Universidad Javeriana de Bogotá a través del proyecto de investigación número 8656 de 2018.

\section{Referencias}

Adam, R., \& Jamieson, C. (2014). Identifying trends in masterplanning: A typological classification system. Urban Design International; Basingstoke, 19(4), 274-290. http://dx.doi.org/10.1057/udi.2013.24

Alexander, C. (1966). A City is not a Tree. Design, 206, 45-55.

Alexander, C., Ishikawa, S., \& Silverstein, M. (1977). A pattern language: Towns, buildings, construction. New York: Oxford University Press.

Blanco, J. (2016). Sustainable development in Chile: paths to introduce sustainability rating systems for neighborhoods. Journal of Architectural and Planning Research, 33(3), 251-270. 
Boyko, C. T., \& Cooper, R. (2011). Clarifying and re-conceptualising density. Progress in Planning, 76(1), 1-61. http://dx.doi.org/10.1016/j.progress.2011.07.001.

Brundtland, G. H. (1987). Our Common Future. New York: Oxford University Press.

Campbell, S. (1996). Green cities, growing cities, just cities? Journal of the American Planning Association, 62(3), 296-312. http://dx.doi.org/10.1080/01944369608975696.

Concejo Colombiano de Construcción Sostenible - CCCS. (2016). Referencial CASA Colombia. Bogotá: CCCS. Recuperado el 9 de abril de 2018, de https://www.cccs.org.co/wp/referencial-casa-colombia/

Congress for the New Urbanism - CNU \& United States Green Building Council - USGBC (2009). LEED for

Neighborhood Development. Washington: CNU. Recuperado el 5 de septiembre de 2019, de https://www.cnu.org/our-projects/leed-neighborhood-development

Congress for the New Urbanism CNU, \& Talen, E. (1999). Charter of the new urbanism (2nd ed.). New York: McGraw Hill Professional.

Dave, S. (2010). High urban densities in developing countries: a sustainable solution? Built Environment, 36(1), 927. http://dx.doi.org/10.2148/benv.36.1.9.

Departamento Administrativo Nacional de Estadística - DANE. (2018). Encuesta Multipropósito (EM) 2017. Bogotá: DANE. Recuperado el 5 de septiembre de 2019, de https://www.dane.gov.co/index.php/estadisticas-portema/pobreza-y-condiciones-de-vida/encuesta-multiproposito/encuesta-multiproposito-2017

Duany, A., Speck, J., \& Lydon, M. (2009). The smart growth manual. New York: McGraw-Hill Education.

Ewing, R., \& Handy, S. (2009). Measuring the unmeasurable: urban design qualities related to walkability. Journal of Urban Design, 14(1), 65-84. http://dx.doi.org/10.1080/13574800802451155.

Farr, D. (2011). Sustainable urbanism: urban design with nature. Hoboken: John Wiley \& Sons.

García, M., Ezequiel, E., Baeriswyl Rada, S., \& Fuentes, P. (2015). Análisis de la sostenibilidad social en tipologías residenciales del gran concepción (CHILE). Cuaderno Urbano, 19(19), 5-28.

Geddes, S. P. (1915). Cities in evolution: An introduction to the town planning movement and to the study of civics. London: Williams \& Norgate.

Gehl, J. (2011). Life between buildings: Using public space. London: Island Press.

Green Building Council Brasil - GBC Brasil. (2017). Check List Certificação GBC Condomínio. Barueri: GBC Brasil. Recuperado el 5 de septiembre de 2019, de https://www.gbcbrasil.org.br/certificacao/certificacaocondominio/documentos/

Jabareen, Y. R. (2006). Sustainable urban forms: their typologies, models, and concepts. Journal of Planning Education and Research, 26(1), 38-52. http://dx.doi.org/10.1177/0739456X05285119.

Jacobs, J. (1961). The death and life of great American cities. New York: Vintage.

Krier, L. (2011). The architecture of community (9th ed.). Washington: Island Press.

Larco, N. (2016). Sustainable urban design - a (draft) framework. Journal of Urban Design, 21(1), 1-29. http://dx.doi.org/10.1080/13574809.2015.1071649.

Luederitz, C., Lang, D. J., \& Von Wehrden, H. (2013). A systematic review of guiding principles for sustainable urban neighborhood development. Landscape and Urban Planning, 118, 40-52.

http://dx.doi.org/10.1016/j.landurbplan.2013.06.002.

Lynch, K. (1960). The image of the city. Cambridge: MIT Press.

Lynch, K. (1981). Good city form. Cambridge: MIT Press.

McHarg, I. L. (1967). Design with nature. Logan: Turtleback Books. 
Montero-Serrano, J., Bosque-Sendra, J., \& Romero-Calcerrada, R. (2008). Cuantificación y cartografía de la sostenibilidad social a partir de tipologías urbanísticas. In: L. Hernández, \& J. M. Parreño (Eds.), Tecnologías de la información geográfica para el desarrollo territorial (pp. 76-91). Las Palmas de Gran Canaria: Servicio de Publicaciones y Difusión Científica de la ULPGC.

Mumford, L. (1968). The urban prospect: Lewis Mumford. London: Secker and Warburg.

Sharifi, A. (2016). From Garden City to Eco-urbanism: the quest for sustainable neighborhood development. Sustainable Cities and Society, 20, 1-16. http://dx.doi.org/10.1016/j.scs.2015.09.002.

Spirn, A. W. (1984). The Granite Garden: Urban nature and human design. New York: Basic Books.

Spirn, A. W. (2014). Ecological urbanism: A framework for the design of resilient cities (2014). In: F. O. Ndubisi (Ed.), The ecological design and planning reader. (pp. 557-570.). Washington: Island Press. http://dx.doi.org/10.5822/978-1-61091-491-8_50.

UN Habitat (2015). International guidelines on urban and territorial planning. Nairobi: UN-Habitat.

Valencia, L., Patricia, A., \& López Bernal, O. (2012). Conceptualización de un modelo de intervención urbana sostenible Ecobarrios en el contexto latinoamericano de reciente industrialización. Revista de Arquitectura, 14, 116-127.

Verdaguer Viana-Cárdenas, C. (2000). De la sostenibilidad a los ecobarrios. Revista de Estudios Sociales y Sociología Aplicada, (119), 59-78.

Wheeler, S. M. (2008). The evolution of built landscapes in metropolitan regions. Journal of Planning Education and Research, 27(4), 400-416. http://dx.doi.org/10.1177/0739456X08315889.

Wheeler, S. M. (2015). Built landscapes of metropolitan regions: an international typology. Journal of the American Planning Association, 81(3), 167-190. http://dx.doi.org/10.1080/01944363.2015.1081567.

Yigitcanlar, T., Kamruzzaman, M., \& Teriman, S. (2015). Neighborhood sustainability assessment: Evaluating residential development sustainability in a developing country context. Sustainability, 7(3), 2570-2602. http://dx.doi.org/10.3390/su7032570.

Editores: Janaina Camile Pasqual Lofhagen, Pontifícia Universidade Católica do Paraná - PUCPR; Christopher Hawkins, University of Central Florida - UCF.

Recibido: Nov. 23, 2018

Aprobado: Fev. 23, 2019 